\title{
Vaginale Inkontinenzoperation mit
} Vaginalschleimhautstreifen

\begin{tabular}{|l|l|l|}
\hline U. & Baumann \\
\hline C. & Meyer \\
\hline
\end{tabular}

Gynäkologisch-geburtshilfliche Abteilung, Spital Limmattal (Chefarzt: Dr. U. Baumann), Schlieren

Dr. med. U. Baumann, Spital Limmattal, CH-8952 Schlieren (Schweiz)

Die Frühresultate aller bekannten operativen Methoden zur Behandlung der Inkontinenz sind relativ gut, die Spätresultate häufig unbefriedigend. Neben der Behebung der bestehenden Inkontinenz muss bei der angewandten operativen Methode der Rezidivprophylaxe das Hauptaugenmerk geschenkt werden. Als Rezidivprophylaxe bei der vaginalen Diaphragmaplastik streben wir eine derbe Narbenplatte zwischen den medialen Rändern des M. levator ani auf Höhe des Blasenhalses an. Weiterhin gehört eine ausgedehnte und sorgfältige hintere Plastik unter Berücksichtigung des Levators in seinem dorsalen Anteil ebenfalls zur Rezidivprophylaxe.

Die Diaphragmaplastik wird in der üblichen Weise durchgeführt. Wir stellen dann den medialen Rand des M. pubococcygeus nahe an seinem Ansatz an der Hinterfläche des Os pubis dar und ziehen einen Streifen von Vaginalschleimhaut durch einen Schlitz des Levatorrandes beidseits. Der Schleimhautstreifen liegt dreifach zwischen den Muskelrändern und wird ohne Zug fixiert. Die hintere Plastik wird sehr ausgedehnt ausgeführt unter Darstellung der Levatoren die in der Medianlinie vereinigt werden. Der Vaginalschleimhautstreifen heilte in bis jetzt über 30 Fallen problemlos ein. Bei der Nachkontrolle von 17 Fallen 5-15 Monate nach der Operation sind 16 Frauen völlig kontinent. 